Neurosurg Focus 23 (4):E13, 2007

\title{
Primary intraosseous meningioma
}

\author{
James B. Elder, M.D., ${ }^{1}$ Roscoe Atkinson, M.D., ${ }^{2}$ Chi-Shing Zee, M.D., ${ }^{3}$ \\ and Thomas C. Chen, M.D., Ph.D. ${ }^{1}$ \\ Departments of ${ }^{\prime}$ Neurosurgery, ${ }^{2}$ Pathology, and ${ }^{3}$ Radiology, University of Southern California, \\ Los Angeles, California
}

\begin{abstract}
$\checkmark$ Primary intraosseous meningiomas are a subtype of primary extradural meningiomas and constitute fewer than $2 \%$ of meningiomas overall, but they represent approximately two thirds of all extradural meningiomas. These types of meningiomas originate within the bones of the skull and thus can have a clinical presentation and radiographic differential diagnosis that is different from those for intradural meningiomas.

Primary intraosseous meningiomas are classified based on their location and histopathological characteristics. Treatment primarily involves resection with wide margins if possible. Very little literature exists regarding the use of adjuvant therapies such as radiation and chemotherapy for these tumors. In fact, the literature regarding primary intraosseous meningiomas consists mostly of clinical case reports and case series. This literature is reviewed and summarized in this article. (DOI: 10.3171/FOC-07/10/E13)
\end{abstract}

\section{KEY WORDS - extradural meningioma - primary intraosseous meningioma • resection}

I NTRAOSSEOUS meningiomas are relatively rare cranial lesions. Their clinical and radiographic presentation guides preoperative diagnosis and operative planning. Future work is necessary to optimize adjuvant therapy for patients whose lesions are not completely resectable. Most meningiomas are considered primary intradural lesions and are located in the subdural space. By contrast, extradural meningiomas arise in locations other than the dura mater, such as the skin, nasopharynx, or neck. ${ }^{13,19}$ Extradural meningiomas constitute 1 to $2 \%$ of all meningiomas. ${ }^{25}$ "Primary intraosseous meningioma" is a term used to describe a subset of extradural meningiomas that arise in bone. ${ }^{28}$ This type of meningioma represents approximately two thirds of all extradural meningiomas. ${ }^{19}$ The vast majority of intraosseous meningiomas arise in cranial bones, although a few cases in which the tumor originated in the mandible have been reported. ${ }^{21}$

This article reviews the radiographic and clinical findings of patients with primary intraosseous meningiomas. For the sake of clarity, the term "intraosseous meningioma" will be used throughout the article and refer to tumors of the skull. Differential diagnosis, nomenclature, and treat-

Abbreviations used in this article: $\mathrm{CT}=$ computed tomography; $\mathrm{MR}=$ magnetic resonance. ment options are discussed. The literature regarding these tumors is reviewed, and two clinical cases from the authors' institution are presented.

\section{Nomenclature and Classification}

Until recently, a unifying classification scheme for meningiomas that arise in locations other than the dura has been lacking. In the literature, meningiomas that arise in locations other than the subdural compartment are given a variety of names. These include ectopic, secondary, extracalvarial, cutaneous, extracranial, primary extraneuraxial, and extradural meningiomas. ${ }^{9,19,33}$ Extradural meningiomas that arise in the skull have been referred to as calvarial, intradiploic, and intraosseous. ${ }^{7}$ Another difficulty with nomenclature in the literature is that primary intradural meningiomas that grow through the bone or metastasize have occasionally been misnamed for their secondary location.

A recent case series offered a unifying nomenclature to reduce confusion caused by the variety of terms present in the literature ${ }^{19}$ The term "primary extradural meningioma" differentiates tumors that arise separately from the dura from those that originate in the dura but have an extracranial extension. This name also differentiates these tumors from extracranial meningiomas that represent distant me- 
tastases from primary intradural meningiomas. Another issue arises when cells from a presumed extradural meningioma invade the dura mater., ${ }^{2,33}$ Depending on the radiographic appearance and surgical findings, these tumors may be difficult to classify definitively. ${ }^{1}$ Some authors state that localization of the mean tumor mass within the skull allows determination of the site of origin of the tumor and designation of an intraosseous meningioma or primary extradural meningioma, even in the presence of dural invasion. ${ }^{3}$ Other authors would maintain that dural invasion precludes a diagnosis of intraosseous meningioma. ${ }^{6}$

The following summarizes the classification scheme for primary extradural meningiomas developed by Lang and colleagues: ${ }^{19}$ Tumors that are purely extracalvarial are Type I, purely calvarial tumors are Type II, and calvarial tumors with extracalvarial extension are Type III. Each category is further divided into convexity (C) or skull base (B) subtypes based on their anatomical location. Thus, intraosseous meningiomas could be considered Type II or III primary extradural meningiomas based on whether extracalvarial extension is observed (Table 1).

\section{Clinical Presentation}

Extradural meningiomas, including intraosseous meningiomas, are reported to occur with the same frequency in each sex or with a slight female predominance, unlike intradural meningiomas, which occur twice as frequently in women as in men. ${ }^{33}$ Like intradural meningiomas, extradural meningiomas predominantly occur later in life, with a median patient age at diagnosis in the fifth decade. These tumors have a bimodal incidence peak in patients, with one peak in the second decade, and a second peak during the fifth through seventh decades of life..$^{19}$ The tumors are nearly uniformly solitary, although a small number of case reports have presented patients with two separate lesions. ${ }^{19}$ In one case, an initial diagnosis of fibrous dysplasia was made after CT showed hyperostosis of the temporal and sphenoid bones. Two years later, a repeat CT scan showed the interval development of a separate area of hyperostosis in the occipital bone, and a biopsy procedure was used to diagnose intraosseous meningioma. ${ }^{11}$

The convexity and the skull base are the two primary locations for intraosseous meningiomas. Like many intracranial lesions, the clinical presentation and resulting differential diagnosis depend largely on the size and location of the lesion.

\section{Convexity Intraosseous Meningiomas}

Convexity intraosseous meningiomas most commonly present as slowly growing scalp masses, with possible rela-

TABLE 1

Classification scheme of primary extradural meningiomas*

\begin{tabular}{cll}
\hline \hline Type & \multicolumn{1}{c}{ Description } & Subclassification \\
\hline I & purely extracalvarial & not applicable \\
II & purely calvarial & B (skull base) \\
& & C (convexity) \\
III & calvarial with extracalvarial extension & B (skull base) \\
& & C (convexity) \\
\hline
\end{tabular}

* Adapted from Lang and colleagues, 2000. tionship to a cranial suture. ${ }^{2}$ Common locations include the periorbital region and frontoparietal region. ${ }^{8}$ These are typically firm and painless, with normal overlying skin, and may be detected incidentally. ${ }^{33}$ Neurological signs and symptoms in patients are usually absent; however, presenting symptoms such as neurological deficit, seizures, vomiting, dizziness, hearing loss, tinnitus, headache, and vague sensations in the head are also reported. ${ }^{8,15,37}$ These symptoms may be present for months or years prior to diagnosis.

\section{Skull Base Intraosseous Meningiomas}

Like their convexity counterparts, skull base intraosseous meningiomas are usually slow growing and painless. Their location, however, may cause different symptoms than convexity lesions for months to years prior to diagnosis. ${ }^{7}$ These symptoms may include cranial nerve deficits, such as ophthalmoplegia or visual field problems, or signs and symptoms related to mass effect, such as proptosis or deformity. ${ }^{10}$ Associated findings on an ophthalmological examination may include conjunctival edema and optic atrophy. Lesions involving the nasal cavity or sinuses may present with nasal obstruction or epistaxis. ${ }^{19}$

\section{Radiographic Appearance}

The radiographic appearance of intraosseous meningiomas depends largely on their location and the effects of the tumor on the surrounding bone. The tumors are typically either the osteoblastic or osteolytic subtype, although mixed versions have been reported. This distinction is not only radiographically significant, but also important for predicting outcome, as is noted in the Discussion section later in the paper.

Like intradural meningiomas, osteoblastic intraosseous meningiomas may induce hyperostosis. ${ }^{8}{ }^{81}$ In fact, the majority of intraosseous meningiomas are of this osteoblastic subtype. ${ }^{1,3}$ In these cases, conventional radiographs will demonstrate hyperdensity associated with the lesion, although superimposed bone structures may limit the usefulness of this radiographic modality. Skull radiographs can detect abnormalities in 30 to $60 \%$ of cases of intraosseous meningioma, including hyperostosis, irregular foci of calcification, and atypical vascular markings. ${ }^{6}$ Computed tomography with bone windows shows a focally thickened, hyperdense lesion expanding the calvaria. The tumor is usually hyperdense on a nonenhanced CT scan, ranging from 65 to 85 Hounsfield units, and enhances densely after contrast administration, similarly to intradural meningiomas. ${ }^{6}$ The expansion of bone and a "ground glass" appearance of this type of intraosseous meningioma may appear radiographically similar to fibrous dysplasia. ${ }^{9}$

More rarely, primary intraosseous meningioma may present as an osteolytic skull lesion. ${ }^{1,18,22,26,30,33}$ These lesions can be distinguished from the osteoblastic subtype based on radiographic findings. The lesion may appear hypodense on plain radiographs, similar to other primary lytic calvarial lesions. Rather than the more common CT findings of thickened sclerotic bone, osteolytic lesions typically cause thinning, expansion, and interruption of the inner and outer cortical layers of the skull. ${ }^{1}$ The lesions are similarly hyperdense compared with brain on a nonenhanced CT scan and enhance homogeneously after contrast administration. ${ }^{6,21}$ 
Crawford and colleagues published a report in $1995^{\circ}$ that reviewed the radiographic findings of the 34 published cases of intraosseous meningioma. Radiographic evidence of hyperostosis was noted in 20 cases (59\%), whereas 11 (32\%) showed osteolytic changes in the surrounding bone. Two cases $(6 \%)$ revealed a mixed picture of both osteolysis and hyperostosis. ${ }^{8}$ As of 2007, only 16 cases of the rare osteolytic subtype of intraosseous meningioma had been described in the literature, which represents fewer than $20 \%$ of reported cases overall. ${ }^{1}$

Magnetic resonance imaging findings for both osteolytic and osteoblastic subtypes of intraosseous meningiomas are similar to those for intradural lesions and allow better delineation of tumors that have extracalvarial soft-tissue extension. The tumors are typically hypointense on T1weighted images and hyperintense on T2-weighted images. Prominent homogeneous enhancement after Gd administration is typical. These tumors do not usually exhibit the "dural tail" often found with intradural meningiomas, but Gd enhancement of the underlying dura may be noted. This dural enhancement could be secondary to dural irritation or tumor invasion. ${ }^{2}$

A recent report evaluated angiographic findings of 10 patients with intraosseous meningioma. Consistent findings in a majority of the patients included an enlarged, tortuous feeding artery, a dense tumor blush, and early venous drainage. Cases in the literature in which angiography was performed reveal that branches of the external carotid artery feed the tumors in most patients. ${ }^{6,18,37}$

Although radionuclide scans are usually normal in patients with intraosseous meningiomas, case reports have demonstrated the occasional usefulness of this procedure. ${ }^{8}$ In one case, a scan with technetium-99m diphosphonate was positive, whereas a technetium-99m pertechnetate scan was negative. ${ }^{40}$ Although both agents may demonstrate uptake in meningiomas, the finding in this case report was probably consistent with the greater affinity of a diphosphonate agent for bone lesions, compared to pertechnetate. ${ }^{5,20}$ Bone scintigraphy using technetium-99m diphosphonate has also been used to assess the response of an intraosseous meningioma to adjuvant therapy in a patient with an unresectable lesion. ${ }^{15}$ Finally, bone scintigraphy has been used to demonstrate a tripling in size of an intraosseous meningioma over a 5-year period in a patient followed up using serial whole-body images after undergoing a radical mastectomy for invasive ductal carcinoma. ${ }^{17}$ Interestingly, plain radiographs of the skull and CT scans with contrast enhancement were normal throughout this same 5-year period. A biopsy specimen showed intraosseous meningioma, but the patient refused surgery.

\section{Differential Diagnosis}

Intraosseous meningiomas may appear osteoblastic, osteolytic, or mixed on CT scans and plain radiographs of the skull, and generating a differential diagnosis depends initially on distinguishing among these subtypes. The osteoblastic subtype may be mimicked by other entities that appear as a focal hyperdense skull lesion on CT. These other entities include meningioma en plaque, osteoma, osteosarcoma, Paget disease, and fibrous dysplasia..,16 Further clinical and radiographic information help narrow the differential diagnosis. For example, fibrous dysplasia may be distinguished from intraosseous meningioma based on the patient's age. Fibrous dysplasia usually stops growing after puberty, whereas intraosseous meningiomas typically appear after puberty and continue to grow slowly. Also, fibrous dysplasia does not have the tumor blush on angiography that is typical of meningiomas. ${ }^{6}$ Meningioma en plaque may induce significant hyperostosis in the overlying bone, and tumor enhancement may be mistaken for a dural reaction to an overlying intraosseous meningioma. The characteristic MR imaging appearance of the intraosseous meningioma, demonstrating homogeneously dense Gd enhancement of the tumor within the skull, may help distinguish this lesion from meningioma en plaque, as well as from other osteoblastic skull lesions such as osteoma (nonenhancing), osteosarcoma (irregular contours, heterogeneous signal, and enhancement) and Paget disease (heterogeneous signal, nonenhancing). Laboratory tests such as serum alkaline phosphatase, which is typically elevated in patients with Paget disease, may assist with the preoperative diagnosis.

The osteolytic subtype of intraosseous meningioma requires a differential diagnosis for solitary osteolytic skull lesions such as hemangioma, chondroma, chondrosarcoma, dermoid, epidermoid tumor, brown tumor, multiple myeloma, plasmacytoma, giant cell tumor, aneurysmal bone cyst, eosinophilic granuloma, or metastatic cancer. ${ }^{1,8,18,30,33}$ The MR imaging appearance of intraosseous meningiomas allows the lesion to be distinguished from most other osteolytic lesions including hemangioma (nonenhancing), eosinophilic granuloma (nonenhancing), aneurysmal bone cyst (multiloculated with fluid levels), and epidermoid/dermoid (fat contents). Laboratory work in patients with plasmacytoma or multiple myeloma should reveal anemia and an immunoglobulin G kappa band after serum protein electrophoresis. Patients with brown tumor should have clinical and laboratory findings consistent with hyperparathyroidism. Malignant lesions such as metastases, chondrosarcoma, and osteosarcoma are often indicated by clinical factors such as known primary cancer or a rapidly enlarging skull lesion, or by radiographic features such as multiple lesions and ragged margins without sclerosis. As will be discussed later, prompt histological diagnosis is warranted in patients with osteolytic intraosseous meningioma, given the higher likelihood of malignancy compared with the osteoblastic subtype.

\section{Histopathology}

Intraoperative pathological examination of an intraosseous meningioma may be limited, especially in cases where hyperostosis is present, due to the presence of bone throughout the specimen. In these cases, decalcification of the specimen is required prior to histopathological assessment. Microscopic pathology often reveals findings pathognomonic for intradural meningiomas, including psammoma bodies and eosinophilic tumor cells with indistinct borders grouped in clusters and whorls. ${ }^{7,9}{ }^{95}$ The nuclei are typically oval and regular, and nuclear pseudoinclusions may be observed. The bone may appear normal, with replacement of the marrow by fat, fibrosis, and tumor cells. Although the reported histopathology commonly reveals a meningotheliomatous meningioma, ${ }^{8,18,26,37}$ other histological types including microcystic, ${ }^{26}$ psammomatous ${ }^{24}$ transi- 
tional, ${ }^{10,12}$ chordoid, atypical, malignant, and fibroblastic ${ }^{39}$ intraosseous meningiomas have been reported. Authors of one report reviewed 48 cases in the literature and found that the majority (30 cases, $62.5 \%$ ) were of the meningothelial type; transitional (12 cases, $25 \%$ ) was the next most common, followed by fibroblastic (4 cases, $8.3 \%$ ), psammomatous ( 1 case, $2.1 \%$ ) and malignant ( 1 case, $2.1 \%) .{ }^{37}$

Histopathological examination of intraosseous meningiomas may reveal an unusual subtype. For example, a chordoid type of intraosseous meningioma was described that demonstrated regions of eosinophilic cells contained in a mucus-rich matrix. This histopathological pattern was similar to, and could therefore be confused with, chordoma. ${ }^{33}$ Consistent with their intradural counterparts, immunostaining of tumor cells of intraosseous meningiomas is usually positive for vimentin and epithelial membrane antigen, but negative for desmin, cytokeratin, and glial fibrillary acidic protein. ${ }^{7,26}$ Tumor cells may show positive staining for $\mathrm{S} 100$ protein. ${ }^{26}$ Limited cytogenetic analysis is available, although one case report stated that chromosome 22 abnormalities were not found. ${ }^{32}$ In another report, the (MIB-1) staining index was $2.4 \%$, suggesting a low proliferative potential. ${ }^{37}$

Although intraosseous meningiomas are largely described as slow-growing, histologically benign lesions, atypical and malignant histological subtypes have been frequently reported. ${ }^{19}$ Recent studies indicate that intraosseous meningiomas have a higher incidence of malignant features than intradural meningiomas. . $^{14,27,38}$ These malignant features may be indicated by microscopic tumor invasion of underlying dura or overlying soft tissue structures. ${ }^{18}$ Other features possibly consistent with a more malignant subtype include increased mitotic activity, increased cellularity, atypia, papillary features, giant cells, and focal areas of necrosis. Metastasis, although rare, also indicates a more malignant subtype. One report reviewed 65 published cases of intraosseous meningioma assessed during the CT era and found that $17(26 \%)$ had atypical or malignant histological features. ${ }^{19}$ Osteolytic lesions, although a more rare form of intraosseous meningioma, have a higher likelihood of atypical or malignant features compared with osteoblastic tumors. Ramifications of this observation are further discussed in later sections. Finally, one reported lesion, characterized histologically as atypical, was found to secrete carcinoembryonic antigen. ${ }^{27}$

\section{Treatment}

Wide surgical excision of an intraosseous meningioma is the treatment of choice and is potentially curative if the surgery is possible. ${ }^{8,15}$ Ideally, cranial reconstruction should occur as part of the same procedure..$^{33}$ Total resection of skull base lesions may not be possible, in which case decompression of vital neural structures is performed. The use of neuronavigation for preoperative planning and intraoperative surgical navigation may facilitate maximal resection of the tumor. Additionally, preoperative assessment of potential defect geometry, for example with three-dimensional CT, may allow implantation of a custom-made cranioplasty at the time of resection. ${ }^{36}$ Use of angiography to rule out vascular involvement has been reported, although embolization was not performed. ${ }^{9}$
Maximal tumor resection and cranial remodeling are important aspects of treatment for intraosseous meningiomas. Other surgical concerns are not necessarily specific for this type of tumor. For example, if dura is resected due to possible tumor involvement, steps must be taken to minimize the likelihood of postoperative cerebrospinal fluid leak. Also, depending on the location of the tumor, more than one surgical approach may be required. ${ }^{10}$ For example, tumors that originate in the orbital walls or frontal bone that extend into the intranasal cavity may require both transcranial and transnasal approaches for maximal resection. ${ }^{7}$

Postoperative imaging is important for evaluating the extent of resection and monitoring for tumor recurrence or progression. Although most of these tumors are histologically benign, they may encroach on or involve certain parts of the skull that render the lesion not completely resectable. Patients with tumors that cannot be completely resected and that are histologically benign and neurologically asymptomatic may be followed up using serial imaging. ${ }^{8}$ Also, as discussed previously, $26 \%$ of these tumors may show evidence of atypical or malignant changes. Patients with such lesions may be more strongly considered for adjuvant therapy, depending on clinical circumstances. Adjuvant therapy may be considered in cases in which patients have unresectable tumors causing neurological deficit or demonstrating malignant or atypical features histologically. This adjuvant therapy may include radiation therapy such as external beam or Gamma Knife surgery, chemotherapy, and bisphosphonate therapy.

Investigators in previous publications recommended adjuvant radiation therapy for patients with lesions that were not completely resectable and in whom the residual lesion is symptomatic or shows radiographic evidence of progression. ${ }^{8,19,27}$ Other case reports describe postoperative radiotherapy for these lesions without indicating the extent of tumor resection; ${ }^{11}$ specific radiation therapy doses and modalities were not usually discussed. Similarly, chemotherapy for metastatic malignant intraosseous meningiomas has been reported, but the exact regimen and medication were not noted. ${ }^{19}$

A recent report described the use of etidronate disodium to treat an inoperable intraosseous meningioma conclusively identified using a biopsy specimen. The tumor involved the sagittal sinus and was associated with significant hyperostosis. The patient received $200 \mathrm{mg}$ /day of etidronate for 6 months, resulting in amelioration of the patient's presenting symptom (tinnitus) and reduction in tracer accumulation on serial bone scintigraphy studies. ${ }^{15}$

\section{Discussion}

A review of the literature reveals fewer than 150 reported cases of intraosseous meningioma. An article in 1995 presented the 36th case in the literature, whereas a publication 5 years later reviewed 123 cases of extradural meningioma originating in the calvaria. ${ }^{8} 19$ Rather than a higher incidence rate, this increase in published cases is likely to represent a combination of improved histopathological capabilities such as immunostains, increased penetrance of radiographic techniques such as MR imaging, and better accuracy in classifying the tumors based on their origin in the skull. Despite this recent surge in published cases, a 

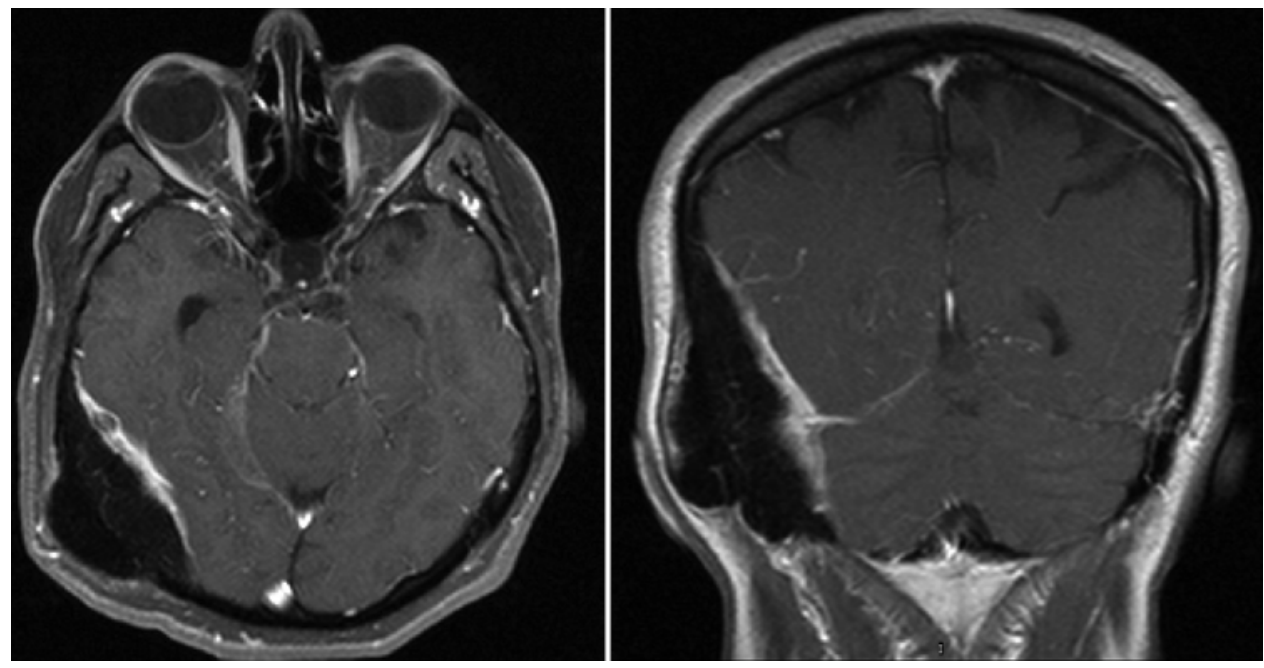

FIG. 1. Representative axial (left) and coronal (right) T1-weighted MR images after Gd contrast administration. The mass is hypointense and based in the right occipital bone. The mass does not enhance after Gd administration, although the dura adjacent to the lesion does.

number of issues regarding intraosseous meningiomas remain incompletely elucidated.

Different theories exist regarding the origin of intraosseous meningiomas. In general, the meninges are derived from mesenchymal cells. Thus, extradural meningiomas could arise in numerous unusual locations as a result of aberrant differentiation and/or misplacement of multipotent mesenchymal stem cells. ${ }^{23,31}$

Alternatively, extradural tumors may arise from cells that become misplaced after differentiation into meningocytes or arachnoid cap cells. For example, arachnoidal cells on blood vessels or nerves traversing the skull may explain the origin of some intraosseous meningiomas. ${ }^{15}$ Cellular dedifferentiation within the skull could also possibly explain the formation of an intraosseous meningioma. ${ }^{7}$ Some authors observed a possible trend for intraosseous meningiomas to be found predominantly near previous fracture sites or at suture lines, most commonly the coronal or pterion sutures. ${ }^{6,829}$ One hypothesis is that these tumors originate from arachnoid cap cells trapped in the cranial sutures, possibly during molding of the cranium at birth., ${ }^{410,35}$ Another theory involves small meningoceles containing arachnoid cap cells becoming trapped within skull fractures or sutures as a result of trauma. ${ }^{1,34}$ Recently published case series, however, do not usually support these latter two theories. ${ }^{6,26,37}$ A recent report published in $2000^{19}$ reviewed 168 cases of extradural meningiomas reported in the literature, of which 114 were calvarial. Only $14(8 \%)$ of the 168 cases were associated with a cranial suture. A separate report reviewed 36 cases in the literature in $1995^{\circ}$ and found that only $5(14 \%)$ were associated with a history of head trau$\mathrm{ma}$ in the region of the tumor. Given the small number of cases and the wide variety of postulated origins of these tumors, it appears unlikely that one theory will fit all cases. Instead, explanations such as head trauma, abnormal cranial molding or embryogenesis, and arachnoid cells accompanying blood vessels or cranial nerves as they traverse the skull could each offer a potentially valid explanation for the origin of an intraosseous meningioma depending on the clinical and radiographic presentation.
As previously mentioned, difficulties with nomenclature discrepancies in the literature were addressed in a recent report by Lang and associates. ${ }^{19}$ Meningiomas that did not originate in the dura were designated "primary extradural meningiomas," and further categorized based on calvarial involvement. Using this system, intraosseous meningiomas are classified as Type II or III primary extradural meningiomas based on the absence or presence of extracalvarial extension, respectively. Each type is then categorized based on whether the tumor is located in the skull base (B) or convexity $(\mathrm{C})$. This system is primarily an anatomical or radiographic classification system, which the authors showed was correlated with clinical outcome. The authors reviewed 96 cases of extradural meningiomas in the literature during the CT era, as well as nine cases from their institution, which all had long-term follow-up data available. Benign intraosseous meningiomas of the skull base with or without extracalvarial extension (Type IIB or IIIB) recurred in $26 \%$ of patients, whereas benign convexity lesions (Type IIC or IIIC) never recurred $(\mathrm{p}<0.05)$ after resection..$^{19}$ Furthermore, the death rate associated with atypical or malignant tumors was $29 \%$ compared with $4.8 \%$ for benign tumors ( $\mathrm{p}$ $<0.004)$. In one patient whose tumor had malignant histopathological features, the tumor metastasized.

Additional consideration could be given for further classifying intraosseous meningiomas based on the radiographic changes observed in the bone involved by the tumor. Specifically, distinguishing between osteoblastic lesions (which are associated with hyperostosis) and osteolytic lesions may improve correlation of the lesions with clinical outcome. Authors of previous reports suggested that intraosseous meningiomas that presented with the clinical and radiographic picture of scalp swelling, osteolytic skull lesion, and extracranial soft-tissue mass were more likely to be malignant meningiomas. ${ }^{24}$ For example, authors of a case series reported on three patients who presented with this clinical triad, all of whom were ultimately diagnosed with malignant meningiomas. ${ }^{38}$ Other case reports also described histologically malignant or atypical intraosseous meningiomas in patients who presented with 


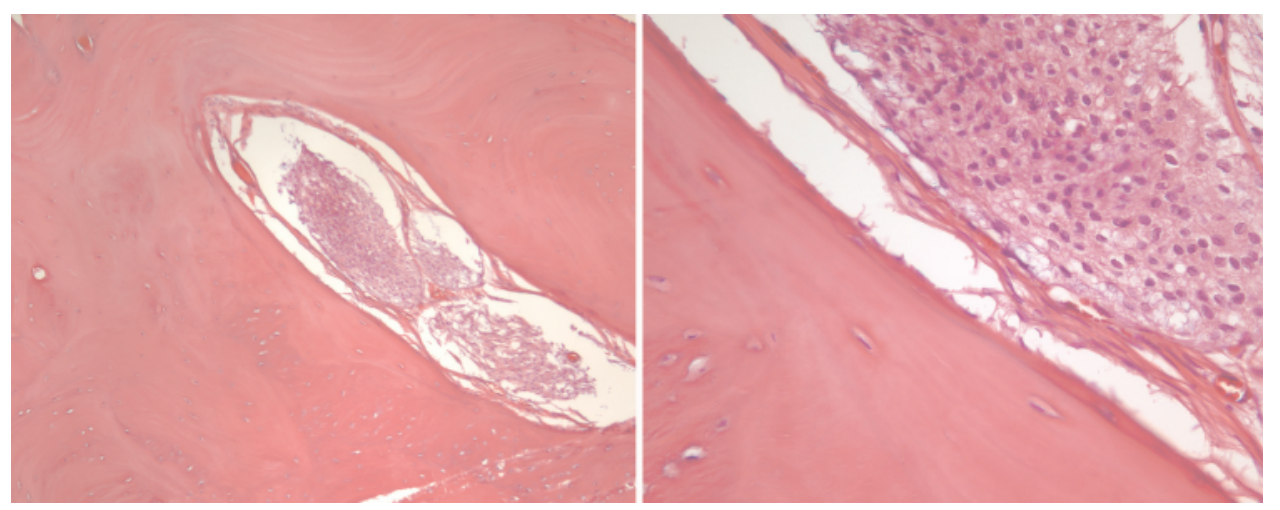

FIG. 2. Photomicrographs showing islands of tumor cells organized in clusters and whorls found amid dense, thickened cortical bone. The tumor cells are cytologically bland, with oval regular nuclei. $\mathrm{H} \& \mathrm{E}$, original magnifications $\times$ 100 left and $\times 400$ (right).

an osteolytic lesion and soft-tissue mass. ${ }^{14,27}$ In one such report, the tumor was noted during resection to invade the dura, arachnoid, and the wall of a large cortical vein. ${ }^{18}$ In general, osteolytic intraosseous meningiomas are more likely to have malignant histopathological characteristics and a more aggressive clinical picture than their osteoblastic counterparts.

Because the majority of these tumors are slow-growing, long-term follow-up is required to rule out recurrent tumors. Recurrence of atypical or malignant intraosseous meningiomas has been reported to occur up to 24 months after resection, whereas histologically benign tumors have been reported to recur up to 120 months after surgery. ${ }^{19}$

\section{Case Reports}

\section{Case 1}

A 41-year-old woman was referred for neurosurgical consultation due to a 2-year history of slowly growing right retroauricular mass. The mass was not tender and no pathological changes in the overlying skin were detected. The patient had no prior medical problems or surgical history, and no history of head trauma. On neurological examination, she showed no deficits.

A CT scan without contrast revealed hyperostosis in the skull corresponding to the region of the retroauricular mass. Magnetic resonance imaging with and without $\mathrm{Gd}$ enhancement showed bone expansion of the right calvaria near the temporal lobe, which was low in signal intensity on all sequences. The T1-weighted images showed adjacent dural enhancement but no enhancement of the lesion after contrast administration. The lesion measured $3 \mathrm{~cm}$ from the inner to outer table and $8 \mathrm{~cm}$ in the cephalocaudad dimension. The underlying brain parenchyma was normal in appearance except for the mass effect exerted by the lesion on the temporal lobe (Fig. 1). Radiographic differential diagnosis included fibrous dysplasia and intraosseous meningioma.

The patient underwent elective craniotomy for resection via a right retrosigmoid incision and exposure. The superior, posterior, and inferior borders of the lesion were identified using neuronavigation. In these three dimensions, the skull was cut $1 \mathrm{~cm}$ away from the tumor border. The anterior border of the tumor abutted the middle ear, and there- fore residual lesion was left behind in this region. Cranioplasty was performed using titanium mesh.

Gross examination by the pathologist revealed a $9.7 \times$ $7.0 \times 2.7-\mathrm{cm}$ irregularly shaped piece of hard skull. The cut surface through an area of thickening revealed a hard, homogeneous, tan-gray surface with no areas of softening or soft tissue in the specimen. The permanent section of the specimen after decalcification showed dense, thickened cortical bone, with expanded Haversian canals and small clusters and whorls of cytologically bland cells with oval regular nuclei (Fig. 2). Immunoperoxidase staining for epithelial membrane antigen was equivocal. Cytokeratin, CD86, and S100 stains were negative. The final histopathological diagnosis was intraosseous meningioma.

The patient experienced no difficulties in the postoperative period. A head CT scan with and without contrast enhancement obtained 3 months after surgery showed persistent hyperostosis of the right occipital bone (Fig. 3). No mass effect or abnormal enhancement was noted. Further surgical or adjuvant treatment, if necessary, will be based on any changes in the appearance of the lesion on future serial images.

\section{Case 2}

A 60-year-old woman was referred for neurosurgical consultation due to a 4-year history of left-eye proptosis, with recent worsening of vision in that eye. She denied any history of head trauma. A CT scan of the head and orbits showed a mass described as proliferation of the left greater wing of the sphenoid bone with a sunburst pattern. The mass protruded into the left orbit, resulting in proptosis (Fig. 4). The differential diagnosis based on this pattern of hyperostosis observed on CT favored intraosseous meningioma, with fibrous dysplasia and osteosarcoma less likely. Magnetic resonance imaging of the brain and orbits with and without Gd enhancement showed a $5.8 \times 4.4 \times$ 3.9-cm extraaxial mass centered in the left sphenoid bone. The mass was centrally hypointense on all imaging sequences, and images revealed periosteal reaction and spiculation, probably secondary to calcium deposits. Also noted were mass effect on the left frontal and temporal lobes and orbital wall, with resulting proptosis (Fig. 5).

Prior to referral to neurosurgery, the patient underwent an elective biopsy procedure of the sphenoid bone by the 


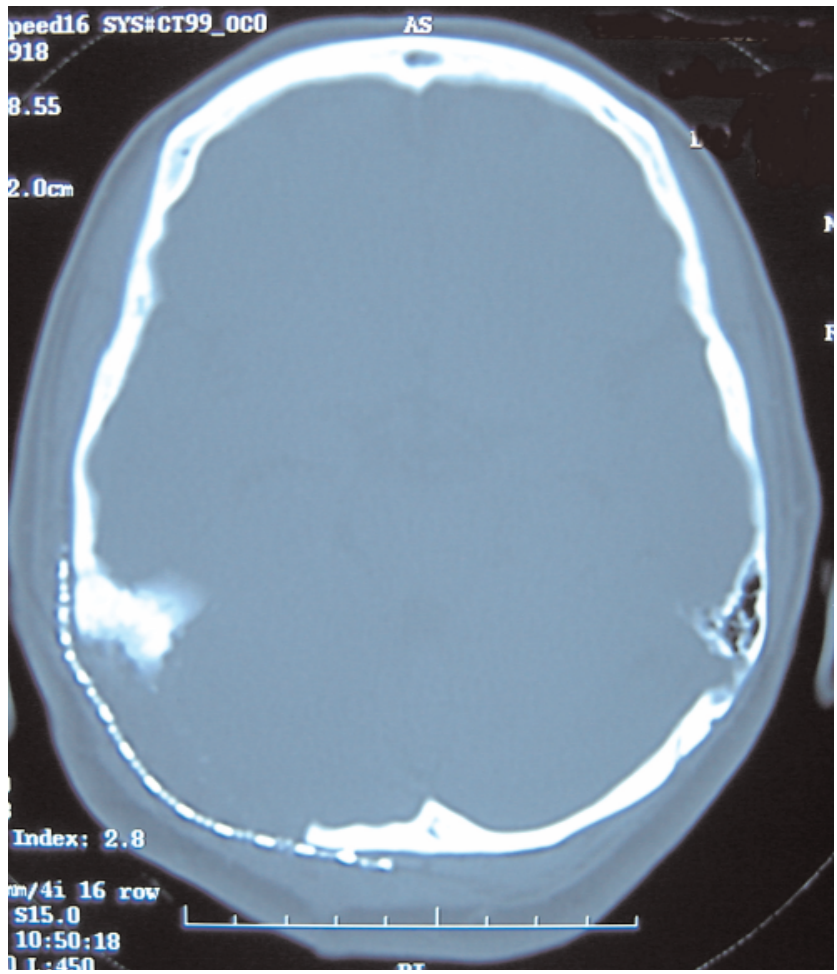

FIG. 3. Case 1. Axial CT scan obtained 3 months after surgery, demonstrating the titanium mesh cranioplasty, as well as residual hyperostosis probably representing residual lesion.

otolaryngology service. The histopathology results indicated an intraosseous meningioma; they specifically showed thickened cortical bone surrounding islands of tumor cells. These cells were bland with oval regular nuclei, and were primarily arranged in a whorl pattern.
The patient underwent a left pterional craniotomy and orbitotomy with subtotal removal of the tumor and orbital decompression. The patient experienced no new neurological problems after surgery. A CT scan of the head and orbits without contrast enhancement performed prior to discharge demonstrated subtotal resection of the tumor. Hyperostosis remained in the left lateral orbital wall and sphenoid. Postoperative MR imaging also showed subtotal resection of the lesion. Follow-up images over the past year have demonstrated a stable appearance of the residual lesion. To date, no adjuvant therapy has been incorporated into the patient's treatment.

\section{Conclusions}

Intraosseous meningiomas are rare lesions that originate in the skull and represent the most common type of extradural meningioma. The lesions are often asymptomatic, but can cause proptosis and neurological symptoms depending on their size and location. Radiographic and clinical presentations generate diagnostic suspicion that may assist with preoperative planning. The majority of these tumors cause hyperostosis that may mimic fibrous dysplasia. Although most are benign, intraosseous meningiomas are more likely to be malignant than their intradural counterparts. The osteolytic subtype of intraosseous meningiomas are more likely to be malignant than the osteoblastic subtype. Intraosseous meningioma should be considered in the differential diagnosis for patients presenting with osteoblastic or osteolytic skull lesions.

It is likely that intraosseous meningiomas originate from entrapment of arachnoid cells within the bone. Multiple theories exist on how the cells become located in the skull, although none are universally accepted and different origins are possible. The treatment of choice is resection, which is potentially curative. Using three-dimensional neuronavigation and operative planning, tumor resection and

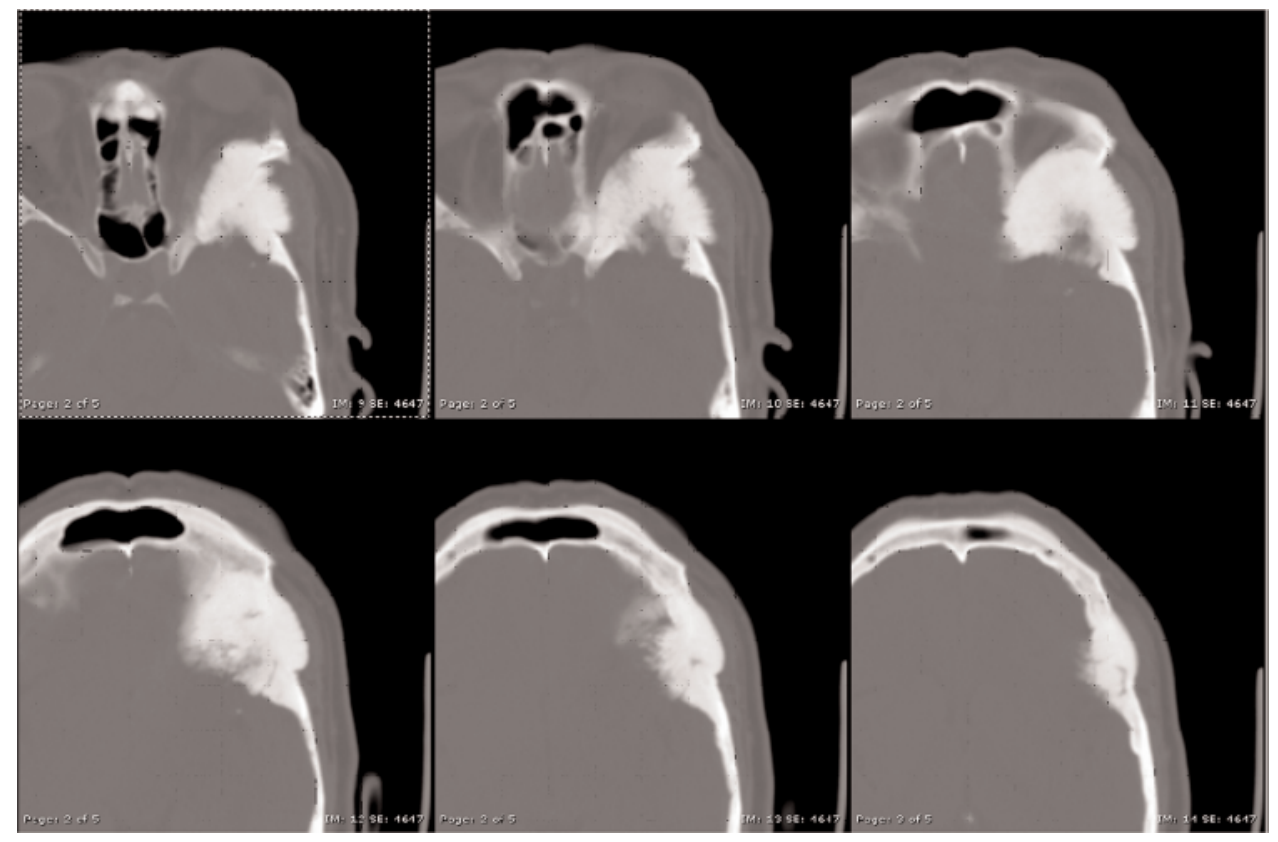

FIG. 4. Case 2. Partial axial views of the preoperative CT scans showing hyperostosis of the left sphenoid bone protruding into the orbit, resulting in proptosis. 

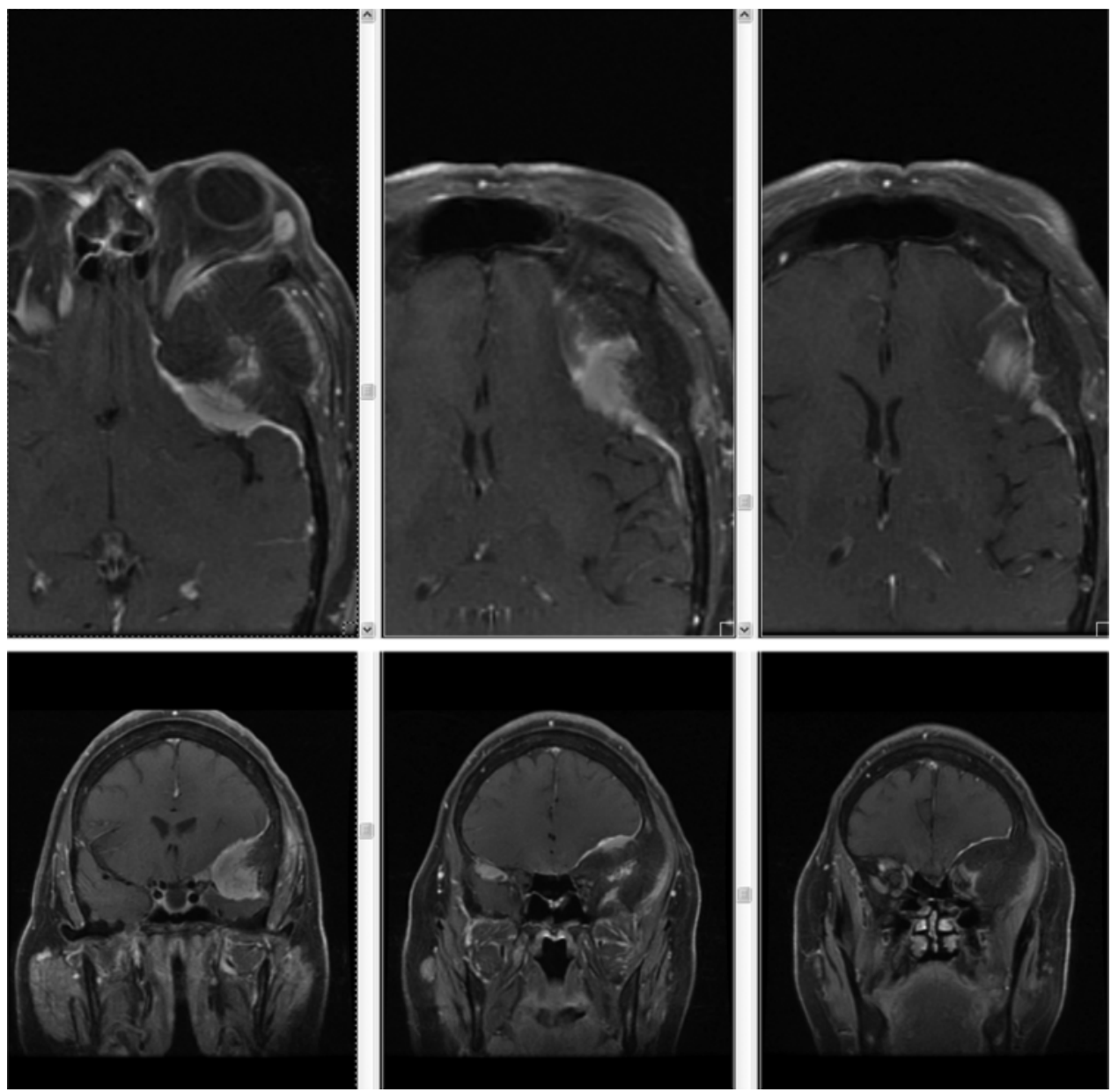

FIG. 5. Case 2. Axial (upper) and coronal (lower) T1-weighted MR images after Gd contrast administration, showing a hypointense mass with moderate peripheral enhancement. Also noted is the mass effect on the orbit causing proptosis.

cranioplasty can be performed simultaneously. Tumors that cannot be completely resected may require adjuvant therapy, which may include radiation therapy, chemotherapy, or bisphosphonate therapy. However, further work is required to elucidate any optimal adjuvant therapy regimens.

\section{References}

1. Agrawal V, Ludwig N, Agrawal A, Bulsara KR: Intraosseous intracranial meningioma. AJNR Am J Neuroradiol 28:314-315, 2007

2. Arana E, Diaz C, Latorre FF, Menor F, Revert A, Beltrán A, et al: Primary intraosseous meningiomas. Acta Radiol 37:937-942, 1996

3. Arana E, Menor F, Lloret RM: Intraosseous meningioma. J Neurosurg 85:362-363, 1996

4. Azar-Kia B, Sarwar M, Marc JA, Schechter MM: Intraosseous meningioma. Neuroradiology 6:246-253, 1974

5. Büll U: The role of nuclear procedures in the diagnosis of intracranial disease. Neurosurg Rev 4:105-122, 1981

6. Changhong L, Naiyin C, Yuehuan G, Lianzhong Z: Primary intraosseous meningiomas of the skull. Clin Radiol 52:546-550, 1997

7. Cirak B, Guven MB, Ugras S, Kutluhan A, Unal O: Frontoorbitonasal intradiploic meningioma in a child. Pediatr Neurosurg 32:48-51, 2000

8. Crawford TS, Kleinschmidt-DeMasters BK, Lillehei KO: Primary intraosseous meningioma. Case report. J Neurosurg 83:912-915, 1995

9. Daffner RH, Yakulis R, Maroon JC: Intraosseous meningioma. Skeletal Radiol 27:108-111, 1998

10. Devi B, Bhat D, Madhusudhan H, Santhosh V, Shankar S: Primary intraosseous meningioma of orbit and anterior cranial fossa: a case report and literature review. Australas Radiol 45:211-214, 2001

11. El Mahou S, Popa L, Constantin A, Jamard B, Cantagrel A, Mazieres B, et al: Multiple intraosseous meningiomas. Clin Rheumatol 25:553-554, 2006

12. Halpin SF, Britton J, Wilkins P, Uttley D: Intradiploic meningiomas. A radiological study of two cases confirmed histologically. Neuroradiology 33:247-250, 1991

13. Hoye SJ, Hoar CS Jr, Murray JE: Extracranial meningioma presenting as a tumor of the neck. Am J Surg 100:486-489, 1960

14. Husaini TA: An unusual osteolytic meningioma. J Pathol 101:57-58, 1970

15. Inagaki K, Otsuka F, Matsui T, Ogura T, Makino H: Effect of etidronate on intraosseous meningioma. Endocr J 51:389-390, 2004

16. Jayaraj K, Martinez S, Freeman A, Lyles KW: Intraosseous meningioma-a mimicry of Paget's disease? J Bone Miner Res 16:1154-1156, 2001

17. Kanmaz B, Weissman DE, Akansel G, Kitapci M, Collier BD: Intraosseous meningioma: appearance on bone scintigraphy over five years. J Nucl Med 34:961-962, 1993 
18. Kudo H, Maeda S, Takamoto T, Tamaki N: Intraosseous meningioma with a dural defect. Neurol Med Chir (Tokyo) 38: 229-231, 1998

19. Lang FF, Macdonald OK, Fuller GN, DeMonte F: Primary extradural meningiomas: a report on nine cases and review of the literature from the era of computerized tomography scanning. J Neurosurg 93:940-950, 2000

20. Lee KT, Tanaka T, Ohi T: Focally accentuated uptake in an area of increased activity in tc-99m HEDP brain scans. J Nucl Med 19:290-291, 1978

21. Lell M, Tudor C, Aigner T, Kessler P: Primary intraosseous meningioma of the mandible: CT and MR imaging features. AJNR Am J Neuroradiol 28:129-131, 2007

22. Levin M, Wertheim SE, Klein E, Lowry J: Unusual lytic intraosseous meningioma. J Neuroimaging 5:247-248, 1995

23. Lopez DA, Silvers DN, Helwig EB: Cutaneous meningiomas-a clinicopathologic study. Cancer 34:728-744, 1974

24. Muthukumar N: Primary calvarial meningiomas. Br J Neurosurg 11:388-392, 1997

25. Muzumdar DP, Vengsarkar US, Bhatjiwale MG, Goel A: Diffuse calvarial meningioma: a case report. J Postgrad Med 47: 116-118, 2001

26. Okamoto S, Hisaoka M, Aoki T, Kadoya C, Kobanawa S, Hashimoto H: Intraosseous microcystic meningioma. Skeletal Radiol 29:354-357, 2000

27. Partington MD, Scheithauer BW, Piepgras DG: Carcinoembryonic antigen production associated with an osteolytic meningioma. Case report. J Neurosurg 82:489-492, 1995

28. Politi M, Romeike BF, Papanagiotou P, Nabhan A, Struffert T, Feiden $\mathrm{W}$, et al: Intraosseous hemangioma of the skull with dural tail sign: radiologic features with pathologic correlation. AJNR Am J Neuroradiol 26:2049-2052, 2005

29. Pompili A, Caroli F, Cattani F, Iachetti M: Intradiploic meningioma of the orbital roof. Neurosurgery 12:565-568, 1983

30. Rosahl SK, Mirzayan MJ, Samii M: Osteolytic intra-osseous meningiomas: illustrated review. Acta Neurochir (Wien) 146: 1245-1249, 2004

31. Shuangshoti S, Netsky MG, Fitz-Hugh GS: Parapharyngeal me- ningioma with special reference to cell of origin. Ann Otol Rhinol Laryngol 80:464-473, 1971

32. Takanashi Y, Mochimatsu Y, Noji M, Yamamoto I: [Multiple meningioma associated with intraosseous and tentorial meningioma - case report.] No To Shinkei 46:884-888, 1994 (Jpn)

33. Tokgoz N, Oner YA, Kaymaz M, Ucar M, Yilmaz G, Tali TE: Primary intraosseous meningioma: $\mathrm{CT}$ and MRI appearance. AJNR Am J Neuroradiol 26:2053-2056, 2005

34. Turner OA, Laird AT: Meningioma with traumatic etiology. Report of a case. J Neurosurg 24:96-98, 1966

35. Van Tassel P, Lee YY, Ayala A, Carrasco CH, Klima T: Case report 680. Intraosseous meningioma of the sphenoid bone. Skeletal Radiol 20:383-386, 1991

36. Westendorff C, Kaminsky J, Ernemann U, Reinert S, Hoffmann J: Image-guided sphenoid wing meningioma resection and simultaneous computer-assisted cranio-orbital reconstruction: technical case report. Neurosurgery 60 (2 Suppl):ONSE173-ONSE174, 2007

37. Yamazaki T, Tsukada A, Uemura K, Satou H, Tsuboi K, Nose T: Intraosseous meningioma of the posterior fossa-Case report. Neurol Med Chir (Tokyo) 41:149-153, 2001

38. Younis G, Sawaya R: Intracranial osteolytic malignant meningiomas appearing as extracranial soft-tissue masses. Neurosurgery 30:932-935, 1992

39. Yuge T, Shigemori M, Tokutomi T, Tokunaga T, Kozima K, Yamamoto T, et al: [A case of intraosseous meningioma.] No Shinkei Geka 19:79-82, 1991 (Jpn)

40. Zaritzky D, Cowan RJ: Intraosseous meningioma: an unusual radionuclide presentation. J Nucl Med 18:943-944, 1977

Manuscript submitted July 18, 2007.

Accepted August 20, 2007.

Support for this work was provided by the Marion and Harry Kieper Neurosurgical Research Fund.

Address correspondence to: Thomas C. Chen, M.D., Ph.D., 1200 North State Street, \#5046, Los Angeles, California, 90033. email: tcchen@usc.edu. 